\title{
Grataloup Christian, Introduction à la
} géohistoireIntroduction à la géohistoire

Paris, Armand Colin, collection Cursus, 2015, 224 p.

Jean-Pierre Husson

\section{CpenEdition}

\section{Journals}

Édition électronique

URL : https://journals.openedition.org/geohist/1357

DOI : 10.4000/geohist. 1357

ISSN : 2264-2617

Éditeur

Association française de la Revue de géographie historique

Référence électronique

Jean-Pierre Husson, «Grataloup Christian, Introduction à la géohistoirelntroduction à la géohistoire », Revue de géographie historique [En ligne], Comptes-rendus, mis en ligne le 20 novembre 2015, consulté le 12 juin 2021. URL : http://journals.openedition.org/geohist/1357 ; DOI : https://doi.org/10.4000/ geohist. 1357

Ce document a été généré automatiquement le 12 juin 2021.

\section{(i) $\odot$}

Ce(tte) œuvre est mise à disposition selon les termes de la Licence Creative Commons Attribution Pas d'Utilisation Commerciale - Pas de Modification 4.0 International. 


\title{
Grataloup Christian, Introduction à la géohistoireIntroduction à la géohistoire
}

Paris, Armand Colin, collection Cursus, 2015, 224 p.

\author{
Jean-Pierre Husson
}

\section{RÉFÉRENCE}

Grataloup Christian, Introduction à la géohistoire, Paris, Armand Colin, collection Cursus, 2015, $224 \mathrm{p}$.

1 Christian Grataloup est professeur à Paris 7 et à Sciences Po Paris. C'est un des meilleurs connaisseurs des rouages de la géohistoire, une discipline encore peu pratiquée dans la géographie francophone malgré les nombreux questionnements puis apports qu'elle peut mobiliser afin de produire un diagnostic complet des territoires. Son ouvrage est à la fois court, condensé et didactique. Il entre dans une collection de manuels destinés aux étudiants du cycle de la licence. La complexité du sujet et les préalables requis pour le lire, en comprendre et en assimiler les démarches relèvent du registre du livre érudit.

2 Ce volume est essentiel pour aborder le couple espace-chronologie. La posture de la géohistoire restitue sa place à l'épaisseur du temps, qu'il soit linéaire, continu, rompu ou bifurqué. Ainsi est comprise la lente sécrétion des territoires et de leur partie la plus lisible: les paysages compris dans leur filiation avec le pays et le paysan. Cette démarche est également à associer à la fiabilité de la mémoire des sols et de la topographie, un axe de recherche aujourd'hui largement révolutionné par l'usage du LIDAR. La réflexion menée n'est pas seulement académique. Elle sert encore à se projeter sur les devenirs et scénarios possibles pouvant concerner un territoire soucieux de valoriser son patrimoine et de soigner son image. 
3 L'auteur familiarise le lecteur avec la prise en compte du temps long dans la construction des lieux à partir des empilements successifs nés des aménagements. Ce sujet souligne l'originalité du rapprochement de l'histoire et de la géographie, deux disciplines enseignées conjointement chez nous ou encore au Japon; ce qui fait figure d'exception. Né sous la plume de Fernand Braudel, le néologisme géohistoire sert à lire, comprendre et interpréter l'espace dans ses interactions avec le temps, qu'il soit cyclique, accéléré dans ses rythmes ou infléchi par les choix retenus ou subis par les hommes. L'auteur confirmer la filiation associant géohistoire et métissage pour souligner la fécondité de cette démarche. A l'origine, le sillon de ces recherches a été tracé dès les années Vingt dans les publications des Annales. Ce thème de recherche se retrouve ensuite chez Fernand Braudel, Xavier de Planhol, Jean-Robert Pitte, etc. Le croisement des perspectives temporelles et des échelles spatiales emboîtées est essentiel pour comprendre notre temps et les legs reçus. Les Anglo-Saxons ont mieux compris que nous tout l'intérêt porté par cette démarche. Elle questionne et éclaire les sujets d'actualité, par exemple les modifications climatiques, l'érosion de la biodiversité, la morphologie des villes, les effets de la mondialisation des échanges, etc. Grâce à ces questionnements, les territoires sont éclairés par le supplément de sens amené avec la patine apportée par la durée. Cette rencontre relève également du besoin social. C'est un moyen de préciser la montée en puissance de la patrimonialisation à inventorier, à faire respecter, à réguler pour ne pas tomber dans l'excès et enfin à transmettre.

Dans sa première partie, l'auteur est guidé par un souci didactique qui l'honore. Il explique et accorde une grande place aux définitions et mises au point. Il développe des commentaires étoffés à propos des mots Territoire, Milieu, Espace. Le croisement de ces termes nous éclaire sur l'historicité, un processus abordant à la fois la reproduction et la transformation d'une société pollenisée par les apports de ses voisins, voire ses envahisseurs. Pour illustrer cela, C. Grataloup souligne par exemple les liens étroits qui unissent la répartition des vents et les directions prises par les flux migratoires dans le Pacifique. Une idée force est qu'espace et lieu amènent la retrouvaille de l'horizontal et du vertical (p. 66).

Echelles, situations et moments structurent le second volet du livre (p.79-154). Ces paramètres sont abordés en partant de l'exemple de Madagascar. L'illustration de ce cas montre que les distances ont une histoire nuancée par la rugosité des lieux, la configuration des puzzles et des réseaux. La géohistoire donne des auréoles au temps avec la diffusion des sphères. L'espace et le temps convergent dans le progrès, un principe mis en avant par les hommes des Lumières. La périphérie est souvent l'élément qui est force de proposition pour tisser l'évolution. Le couple temporalité/ spatialité a cru en importance avec les grandes découvertes et leur corollaire : certes l'afflux des métaux précieux, surtout la confrontation des sociétés à racines et à pattes (p. 116). La première favorise l'accumulation des biens alors que la société caravanière vit avec le strict nécessaire. Pour aller plus loin, C. Grataloup souligne qu'il n'y aurait pas de richesse dans le fait géographique sans l'élargissement du système binaire vers le multiscalaire. C'est un avertissement pour se défier de l'Etat-nation monoscalaire, faisant à coup sûr progresser la frontière vers l'affrontement. Les échelles réveillent à la fois l'empire et le poly territoire. Les chapitres ayant traité de l'impossible déterminisme environnemental concluent sur une géographie des historicités résumée dans la formule suivante : Densité+connexité= historicité. 
6 Dans la dernière partie intitulée « Principes géohistoriques ", C. Grataloup insiste sur la relativité des faits sociaux, les récurrences constatées et les efforts entrepris pour caser ces données dans des modèles comparatifs, des scénarios possibles. Pour établir cette démonstration, il mobilise des exemples variés : la chine, nos réseaux urbains actuels et antiques, Constantinople. L'auteur termine sa réflexion sur le couple fertile formé par l'échelle et l'identité, avec des logiques spatiales et des résistances territoriales.

7 Mettre en avant la géohistoire, c'est avant tout replacer l'espace dans un processus historique, un duo espace-temps. Ce dernier sert de trait d'union pour expliquer les espaces dont nous sommes usufruitiers. En terminant sur la fécondité de ce lien, C. Grataloup livre un message très optimiste sur la vitalité de ce secteur de recherche plein de promesses. 